Chapter 8

\title{
Endovascular Treatment of Endoleaks Following EVAR
}

\author{
Zaiping Jing, Qingsheng Lu, Jiaxuan Feng and \\ Jian Zhou \\ Additional information is available at the end of the chapter \\ http://dx.doi.org/10.5772/54836
}

\section{Introduction}

Since the first endovascular aneurysm repair (EVAR) of abdominal aortic aneurysm (AAA) was introduced 20 years ago, work toward reducing the morbidity and mortality has never been halted. Technological advances, as well as increased operator experience, have significantly reduced post-EVAR complications, such as peripheral embolization, postimplantation rupture, graft migration, and contrast-induced nephropathy, et al. However, endoleak - the persistent flow of blood within the aneurysm sac - is one of the major reasons for lifelong imaging surveillance.

Endoleaks are often considered significantly adverse event of EVAR since persistentce of blood flow and pressure in the aneurysm sac. It continues to be a challenge in the endovascular approach to AAA repair. Some of these leaks are related to anatomic factors and patient selection, others are device related, and some (especially type II leaks) seem to be intrinsic to the endovascular procedure. Certain endoleaks require treatment as soon as they are detected to continue to perfuse and pressurize the aneurysm sac, thereby conferring an ongoing risk of aneurysm enlargement and rupture. But the need for treatment of others remains controversial, since the associated increase in intrasac pressure varies depending on the type of endoleak. Using endovascular techniques, the majority of endoleak can be successfully treated, without the need for open surgery. In this chapter, we will introduce the classification, clinical relevance and endovascular management of the endoleaks after EVAR.

\section{Definition of endoleak}

More than 20 years after the procedure was introduced to treat AAA, it is remarkable that certain aspects of endovascular treatment for AAA remain poorly understood. One major issue 
is the occurrence and significance of endoleaks. White et al were the first to systematically describe and classify endoleaks, describing five major categories [1]. And these five types of endoleaks have been described by Ad Hoc Committee for standardized reporting practices of EVAR in the Society for Vascular Surgery/ American Association for Vascular Surgery [2]. There were type I to IV endoleaks, and a supplementary category of "endoleaks of undefined origin" which was defined as flow visualized but source undetermined. And there was a separate adjunctive category of "endotension", which was referred to instances of aneurysm expansion after EVAR without detectable flow in aneurysm sac.

- Type I endoleak is defined as a sealing failure at one of the attachment sites of the graft to the vessel wall (proximal leak, type Ia; distal, type Ib; inadequate seal at iliac occlude plug, type Ic). Arterial flow therefore leaks alongside the graft and into the perigraft space.

- Type II endoleak is defined as retrograde flow through collateral vessels (lumbar artery, inferior mesenteric artery, hypogastric artery, and accessory renal artery et al.) into the the aneurysmal sac.

- Type III endoleak is defined as graft failure. Type IIIa endoleak is referred to flow from module disconnection, and type IIIb is flow from fabric disruption.

- Type IV endoleak is from porous fabric of the stent graft.

A more detailed classification of endoleaks and endotension was introduced by an international consensus of experts [3]. The classification included type I to IV endoleaks, and endotension (also recognized as type $\mathrm{V}$ endoleak) (see Table 1). As experience has been gained, it is clear that, in some cases, an endoleak can involve more than one mechanism.

\begin{tabular}{ll}
\hline Type & \multicolumn{1}{c}{ Description: source of perigraft flow } \\
\hline & Attachment site leaks \\
\hline II & Proximal end of endograft \\
\hline & Distal end of endograft \\
\hline Iliac occlude (plug) \\
\hline III & Branch leaks (without attachment connection) \\
\hline & Simple or to-and-from (from only 1 patent branch) \\
\hline Complex or flow-through (with 2 or more patent branches) \\
\hline Graft defect \\
\hline JV Endotension & Junction leak or modular disconnection \\
\hline & Fabric disruption (midgraft hole) \\
\hline Minor (<2mm; eg. Suture hole) \\
\hline Major (>2mm) \\
\hline Graft wall (fabric) porosity; <30days of placement
\end{tabular}


endoleak

Without endoleak

With sealed endoleak ("virtual endoleak")

With type I or type III endoleak

With type II endoleak

Adapted from Veith FJ, Baum RA, Ohki T, Amor M, Adiseshiah M, Blankensteijn JD, et al. Nature and significance of endoleaks and endotension: summary of opinions expressed at an international conference. J Vasc Surg. 2002 May;35(5): 1029-35. With permission.

Table 1. Classification of endoleaks and endotension after endovascular aortic repair.

Besides the cause of perigraft flow, the endoleaks can be classified according to the time of first detection as perioperative (within 24 hours), early (within 90 days after the primary EVAR) and late (more than 3 months after the primary procedure). And endoleaks can also be described as persistent, transient or sealed, recurrent, successfully treated or unsuccessfully treated.

\section{Type I endoleak}

As this flow is direct and under systemic pressure, it represents a failure in treatment of the aneurysm, which continues to be pressurized and at risk of continued expansion and rupture. Previous study demonstrated that mean pressure index (sac pressure divided by systemic pressure) was varied in the liquid and in the thrombosed sections of the sac after EVAR. They found that type I endoleaks were associated with mean pressure indexes of $93 \%$ in the liquid areas and $62 \%$ in the thrombus [4]. So it is mandatory to correct type I endoleaks whenever possible, as spontaneous resolution over time cannot be expected.

The incidence of type I endoleak is not low, especially for cases with difficult anatomy situations. The incidence has been reported to be more than $10 \%$ of EVAR procedures, with $4.2 \%$ at 30 days, $3.5 \%$ within 1 year, and $6.7 \%$ at 3 year [5].

\section{Proximal type I endoleak (type Ia endoleak)}

When AAA are with short neck (proximal attachment zones $<15 \mathrm{~mm}$ ), large neck (diameter $>32 \mathrm{~mm}$ ), irregular neck (such as cone-shaped neck), uneven neck (calcification or thrombus) and proximal angulations $>60^{\circ}$, the incidence can be higher than the above mentioned [6]. In common circumstances, $20 \%$ oversizing of the proximal endograft attachment and deploying 
the endograft close to the origin of the renal arteries can help to prevent type Ia endoleak and achieve satisfying sealing.

In the pivotal trial period of EVAR, case selection involved adherence to fairly rigorous anatomic criteria, including vessel size, angulation, shape, length of the infrarenal neck, and adequacy of the distal landing zones. With increasing experience and the availability of newer devices, these criteria have been weakened. Nevertheless, there remain certain types of anatomy that are not well suited to EVAR and are best treated with open surgical technique. Proximal attachment zones less than $10 \mathrm{~mm}$, particularly with angulation greater than $60^{\circ}$, present insurmountable problems with the use of currently available devices.

The development of devices more suitable to extreme degrees of angulation, fenestrated and branched devices, and improvement of sealing mechanisms (ie, endovascular stapling) have expanded the use of EVAR. But as the selection criteria of candidates for EVAR have expanded over time, the detection of type Ia endoleak has become more frequent.

The current availability of various proximal extender cuffs (Figure 1) and other modular devices (such as ballooning and bare stent, Figure 2) for dealing with attachment site leaks has allowed most of these leaks to be treated at the time of the initial procedure. Deployment of Palmaz stent (Cordis Endovascular, Johnson and Johnson Co. UK) or sinus-XL Stent (OptiMed, Ettlingen, Germany) to diminish the proximal type I endoleak was introduced [7] (Figure 3).
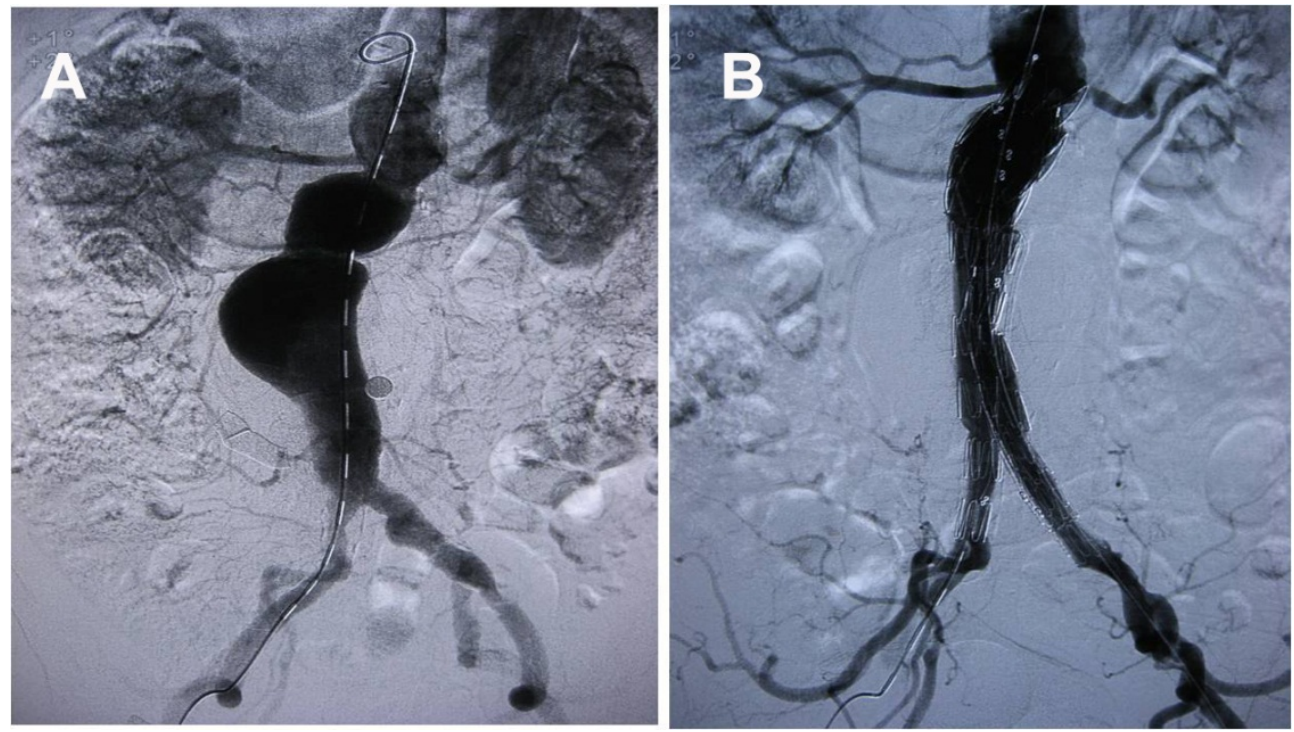

Figure 1. For abdominal aortic aneurysm with saccular neck, adjunctive short-large Cuff is effective to diminish type la endoleak, and realize best proximal fixation. 

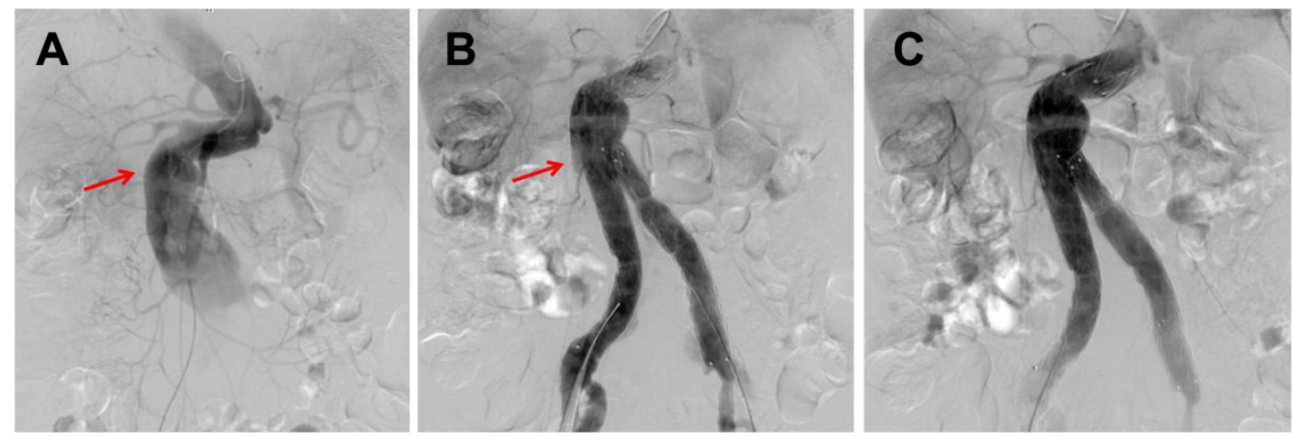

Figure 2. A: Pre-operative angiography revealed the AAA with irregular neck and severe angulation. B: Intra-operative angiography confirmed the significant type la endoleak. C: After proximal ballooning, the endoleak was diminished.
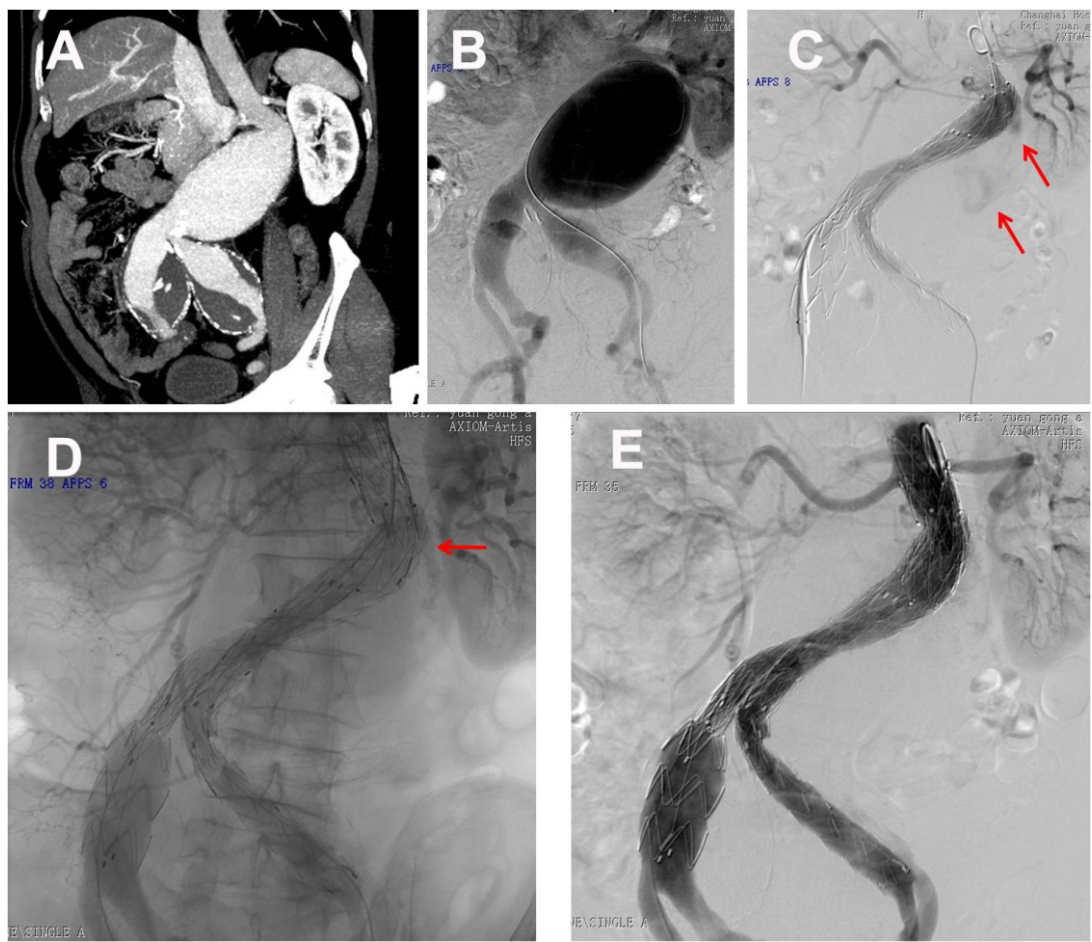

Figure 3. A: Pre-operative CTA revealed AAA, with large aneurism sac (maximal diameter $\approx 90 \mathrm{~mm}$ ) and angulated neck. B: Pre-operative angiography confirmed the large AAA, and bilateral iliac artery aneurysms. C: After the bifurcated endograft and distal extension were deployed, there was remarkable type la endoleak. D: After the proximal cuff and ballooning, the type I endoleak was improved, but still significant. E: Proximal bare stent was deployed (sinus-XL Stent, OptiMed, Ettlingen, Germany)), and the final angiography evidenced the diminished endoleak. 
However, under some circumstances, such as when the proximal edge of the stent-graft is very close to the renal artery, proximal cuffs cannot further extend the proximal fixation zone. If balloon dilation and additional bare stents cannot eliminate the leak alongside the graft fixation zone, then other practical options are needed to resolve the difficulty endoluminally.

\section{Fibrin glue sac embolization to treat type Ia endoleak}

Embolization techniques were introduced for treating type I endoleak $>10$ years ago [8]. The use of microcoils, n-butyl 2-cyanoacrylate adhesive, or other embolic agents (such as Onyx) have been utilized to treat type I endoleaks, but their effectiveness is still unproven $[6,9,10]$. Studies of fibrin glue embolization of endoleak are few and primarily focused on the prevention of type II endoleak [11-13]. So we examined our 5-year experience using fibrin glue to assess the technique's feasibility, safety, and effectiveness in treating type I endoleaks in the intraoperative setting.

\section{Methods}

\subsection{EVAR procedure}

Between August 2002 to February 2009, 783 patients with AAA underwent EVAR at our institution. All patients were evaluated preoperatively with computed tomographic angiography (CTA); appropriate reconstructions from the CT datasets were analyzed on a workstation (Leonardo; Siemens, Erlangen, Germany) to obtain pertinent measurements for endograft sizing ( $20 \%$ oversizing typically used). During the EVAR procedure, endoleaks were identified by angiography and classified as delineated in the current reporting standards [2]. According to our policy, type I endoleaks were treated at the time of diagnosis. If a type I endoleak was still present after proximal balloon dilation and cuff placement, or if a proximal cuff could not be placed because of a short landing zone, a standard procedure was employed to embolize the aneurysm sac using fibrin glue.

\subsection{Embolization technique}

According to our standard EVAR protocol, a 0.035-inch guidewire was positioned in the aneurysm sac before the main body of the stent-graft was deployed. In patients with a persistent type I endoleak after initial closure procedures failed, a 23-mm-long 5-F Brite Tip introducer sheath (Cordis Europe, Roden, The Netherlands) was advanced along the preloaded guidewire into the aneurysm sac. An aneurysmogram was performed to demonstrate the patency of the aneurysm and the flow direction in the aortic branches, such as the lumbar and hypogastric arteries. If no other source of the leak could be found, such as an accessory renal artery arising from the aneurysm or combined types I and II endoleaks, a pressure transducer (Edwards Lifesciences, Irvine, CA, USA) was connected to the 5-F catheter [14]. The intrasac pressure was measured by placing the catheter tip at 3 different locations around 
the nidus of the endoleak. The mean of the 3 pressures was recorded, as were the systolic, diastolic, and mean systemic pressures and the pulse pressure. The mean pressure indexes (MPI, sac pressure divided by systemic pressure) were calculated.

Next, a 25-cm-long, double-lumen Duplocath catheter (Baxter/Hyland Immuno AG, Vienna, Austria) connected to a Duploject Y-connector was introduced into the Brite Tip sheath. To block proximal blood flow when the glue was injected, a balloon was positioned in the proximal native aorta. The 5-mL aprotinin and thrombin solutions (Bei Xiu Biotech Co. Ltd., Guangzhou, PR China) were injected into the aneurysm sac through the 2 syringes of the Duploject Y-connector; the components synthesized fibrin glue in the sac as they mixed. Blocking proximal blood at this time facilitates formation of the clot. After the fibrin glue injection, the intrasac pressure was again recorded to monitor the change caused by embolization. The embolization procedure was repeated until the type I endoleak was eliminated, as evidenced by stable contrast inside the sac, a decrease in the intrasac pressure, and angiography (Figure 4).

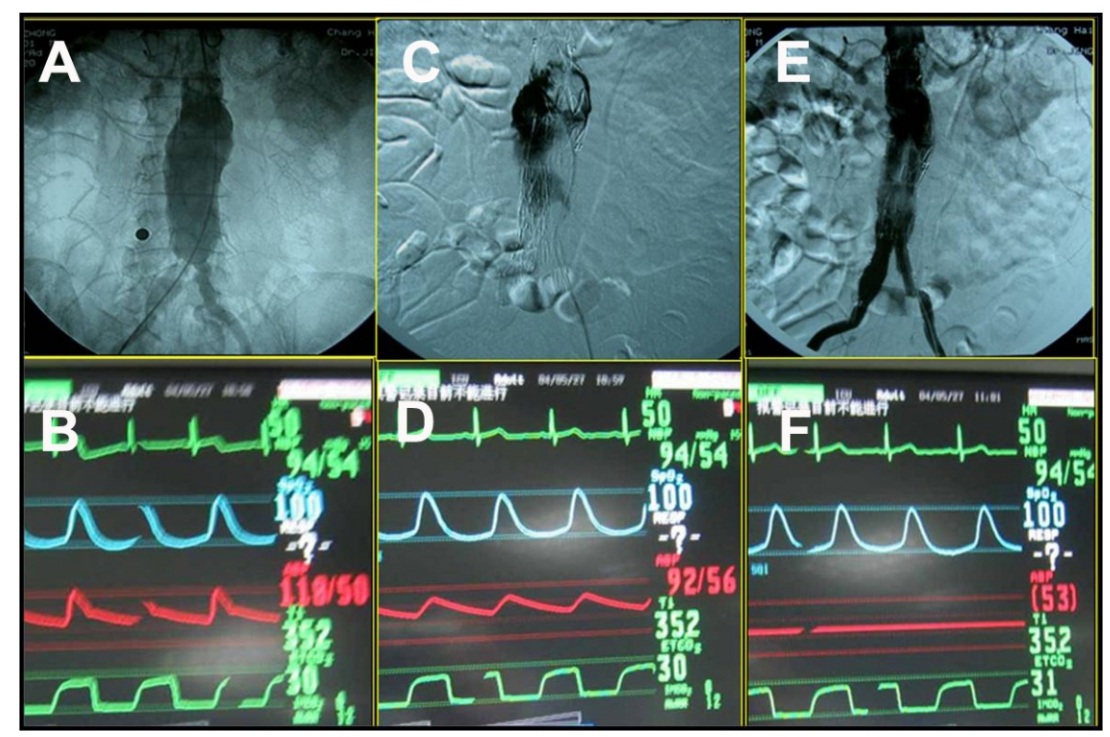

Figure 4. A: Abdominal aortic aneurysm with severe calcified neck. B: The system pressure was revealed by the red wave and number: $118 / 50 \mathrm{mmHg}$. C: The intra-operative angiography after the first endografting revealed type la endoleak. Proximal cuff and ballooning could not eliminate the endoleak. D: The Intra-sac Pressure was measured by pre-placed intrasac catheter. The sac pressure was 92/56 mmHg. E: After intra-sac jel coagulation, the endoleak was eliminated. F: The intra-sac pressure decreased. The amplitude of the systolic-diastolic waveform was reduced significantly after the fibrin glue embolization, which was considered as a sign of diminish of endoleak.

In follow-up, CTA was performed at 3, 6, and 12 months and annually thereafter. Maximum aneurysm diameter and the presence of endoleaks were recorded. A $>2$-mm decrease in sac diameter was considered significant. The endpoints of follow-up were death and severe 
ischemic events, such as paraplegia, ischemic intestinal obstruction, renal artery occlusion, and lower limb ischemia.

\subsection{Patient sample}

A retrospective review of records and our department's vascular database was conducted to identify all patients who underwent fibrin glue sac embolization for type I endoleak. The search found $42(5.4 \%)$ patients (37 men; mean age $73 \pm 8$ years, range $51-88$ ) who had persistent type I endoleaks treated intraoperatively with intrasac fibrin glue injection. Patient and aneurysm characteristics are given in Table 1 . The mean maximal aneurysm diameter was $59.5 \pm 14.7 \mathrm{~mm}$ (range $41-100$ ). The mean diameter of the proximal neck was $21.4 \pm 4.5 \mathrm{~mm}$, and the mean length was $22.7 \pm 14.7 \mathrm{~mm}$; notably, 16 (38.1\%) patients had proximal necks $<10$ $\mathrm{mm}$ long. Five $(11.9 \%)$ patients had proximal neck angulation $>60^{\circ}$.

\begin{tabular}{|c|c|}
\hline \multicolumn{2}{|c|}{ Patient characteristics } \\
\hline Age, y & $73.31 \pm 8.35$ \\
\hline Men & $37(88.1 \%)$ \\
\hline Comorbidities & $38(90.5 \%)$ \\
\hline Hypertension & $28(66.7 \%)$ \\
\hline Hyperlipidemia & $8(19.0 \%)$ \\
\hline Diabetes & $3(7.1 \%)$ \\
\hline Urinary system diseases* & $8(19.0 \%)$ \\
\hline Coronary heart disease & $8(19.0 \%)$ \\
\hline Pulmonary diseaset & $7(16.7 \%)$ \\
\hline Tobacco use ("/>20 years) & $27(62.3 \%)$ \\
\hline \multicolumn{2}{|l|}{ Aneurysm characteristics } \\
\hline Maximal aneurysm diameter, $\mathrm{mm}$ & $59.5 \pm 14.7$ \\
\hline Aneurysm length, mm & $84.1 \pm 30.4$ \\
\hline Proximal neck diameter, $\mathrm{mm}$ & $21.4 \pm 4.5$ \\
\hline Proximal neck length, $\mathrm{mm}$ & $22.7 \pm 14.7$ \\
\hline Proximal neck length $<10 \mathrm{~mm}$ & $16(38.1 \%)$ \\
\hline Proximal neck angulation & $38.5 \pm 30.7$ \\
\hline Proximal neck angle "/>60 & $5(11.9 \%)$ \\
\hline
\end{tabular}

Continuous data are presented as means \pm standard deviation; categorical data are given as counts (percentages).

${ }^{*}$ Chronic renal failure, cyst, urinary stone, renal artery stenosis.

tChronic obstructive pulmonary disease, tuberculosis.

Table 2. Patient and Aneurysm Characteristics 
The most commonly used stent-graft was the Talent model (Medtronic Vascular, Santa Rosa, CA, USA) in 32 (76.2\%) patients, following by the Zenith (Cook Inc., Bloomington, IN, USA) in $6(14.3 \%)$, and the Aegis (MicroPort Medical, Shanghai, People's Republic of China) in 4 (9.5\%). Twenty ( $47.6 \%$ ) patients had more than 1 stent-graft deployed to exclude the aneurysm. The mean oversizing rate was $21.2 \% \pm 12.0 \%$ in this cohort.

\subsection{Statistical analysis}

Parametric data are presented as mean \pm standard deviation; nonparametric data (e.g., followup time) are given as median and range. Survival analysis was performed using the KaplanMeier method; comparisons were examined using a paired sample $t$ test. A threshold of 0.05 was used for statistical significance.

\section{Results}

In the 42 patients with type I endoleak resistant to first-line procedures (balloon dilation and stent/cuff placement), 22 additional devices (8 stents, 14 cuffs) were placed in the initial attempts to resolve the endoleak. After fibrin glue injection, 41 (97.6\%) of the 42 endoleaks were resolved with a mean $15 \pm 10 \mathrm{ml}$ of glue. Nearly half of the patients $(20,47.6 \%)$ received $\leq 10 \mathrm{~mL}$ of glue; 5 (11.9\%) patients needed $>30 \mathrm{ml}$. One endoleak persisted despite proximal cuff implantation and the use of $30 \mathrm{ml}$ of glue.

Systolic, diastolic, mean, and pulse pressures and the MPI were all significantly decreased after fibrin glue embolization, especially the pulse pressure (Table 2). The amplitude of the systolicdiastolic waveforms of the 41 successfully treated patients were reduced significantly after the fibrin glue embolization, which was considered as a sign of diminish of endoleak.

\begin{tabular}{|c|c|c|c|c|c|c|}
\hline \multirow[b]{2}{*}{ Pressure } & \multicolumn{2}{|c|}{ Pre-embolization } & \multicolumn{2}{|c|}{ Post-embolization } & \multicolumn{2}{|c|}{ Decrease } \\
\hline & $\begin{array}{c}\text { Sac Pressure, } \\
\mathrm{mmHg}\end{array}$ & MPI, \% & $\begin{array}{c}\text { Sac Pressure, } \\
\mathrm{mmHg}\end{array}$ & MPI, \% & $\begin{array}{c}\text { Sac Pressure, } \\
\mathrm{mmHg}\end{array}$ & MPI, \% \\
\hline Systolic & $119.3 \pm 18.6$ & $92.2 \pm 5.3$ & $44.7 \pm 9.4$ & $34.1 \pm 6.2$ & $74.5 \pm 12.1^{*}$ & $57.9 \pm 4.2^{*}$ \\
\hline Diastolic & $70.3 \pm 9.3$ & $79.5 \pm 7.6$ & $38.7 \pm 5.9$ & $43.2 \pm 6.7$ & $31.7 \pm 5.7^{\star}$ & $27.0 \pm 5.1^{*}$ \\
\hline Mean & $86.6 \pm 12.2$ & $87.8 \pm 4.4$ & $40.7 \pm 6.9$ & $35.6 \pm 6.4$ & $46.0 \pm 7.5^{\star}$ & $51.2 \pm 3.2^{*}$ \\
\hline Pulse & $48.8 \pm 10.3$ & $124.1 \pm 8.4$ & $6.0 \pm 4.0$ & $15.3 \pm 9.5$ & $42.8 \pm 8.0^{*}$ & $108.5 \pm 6.2^{*}$ \\
\hline
\end{tabular}

Data are presented as means \pm standard deviation.

MPI: mean pressure index.

${ }^{*} \mathrm{p}<0.05$.

Table 3. Comparison Between Pre- and Post-embolization Intrasac Pressures 
No patient exhibited an allergic response to fibrin glue injection, but 1 (2.4\%) patient developed right lower extremity ischemia, which was related to wire/catheter manipulations rather than the fibrin glue treatment itself. The 80-year-old patient who had a failed fibrin glue attempt was converted to open surgery; he died in the Intensive Care Unit 2 months later from multiorgan dysfunction syndrome. Two patients who were successfully treated by fibrin glue injection succumbed to myocardial infarction within 5 days of the EVAR procedure (30-day mortality $4.8 \%$ ).

Over a median follow-up of 39.9 months (range 10-88), the surviving patients complied with the surveillance protocol without loss to follow-up. Three patients died; 1 death was due to progression of an untreated AAA and the others to causes unrelated to aortic aneurysm disease. Cumulative survival was $90.5 \%$ at 1 year, $87.0 \%$ at 3 years, $82.6 \%$ at 5 years.

The mean maximal aneurysm diameter at the latest follow-up visit in 39 patients was $49.0 \pm 11.6$ $\mathrm{mm}$, significantly different from the $59.5 \pm 14.7 \mathrm{~mm}$ preoperative maximal aneurysm diameter $(\mathrm{p}<0.001)$. Postoperative maximal aneurysm diameters decreased in 25 patients and were stable in 10 patients. Of the 4 patients with increased aneurysm diameter detected during follow-up, a 73-year-old patient had a 22-mm increase after 44 months owing to a type IV endoleak; this patient was converted to open surgery. Two other patients (81 and 77 years of age) had diameter increases of $23 \mathrm{~mm}$ after 4 months and $6 \mathrm{~mm}$ after 34 months, respectively, but no blood flow within the aneurysm sac was found by CTA. Considering their advanced age, these patients are being closely followed. The last patient had a 20-mm increase after 4 months; he died of renal failure brought on by renal artery compression from the enlarged aneurysm, but no endoleak could be found. No stent-graft dislocation, fracture, or other complication was found in the follow-up period; in particular, no recurrence of type I endoleak was revealed by follow-up CTA.

\section{Discussion}

Various proximal extender cuffs and other adjuncts, such as balloon molding and bare stents, can deal with most of type I endoleaks once detected. However, when no sufficient landing zone is available proximally, the potential to occlude the renal arteries precludes the deployment of a proximal cuff. In some cases, even proximal balloon dilation and extension cuff implantation cannot resolve the type I endoleak. Under these circumstances, catheter-based procedures, including glue and/or coil deposition, can be performed $[9,10]$. Maldonado et al. reported the success rates for several methods of treating type I endoleaks: n-butyl cyanoacrylate $92.3 \%$, extender cuffs $80 \%$, and coils with or without thrombin $75 \%$. Microcoil embolization can be laborious and time-consuming. Moreover, when numerous coils are deployed, the financial burden is too great for our patients without medical insurance, so we employed fibrin glue. In our study, which had a larger sample and longer follow-up, $98 \%$ of the type I endoleaks were eliminated by transcatheter fibrin glue embolotherapy, superior to the other methods tested so far. 
Fibrin glue is a non-cytotoxic, fully resorbable biological adhesive matrix. The 2 main components are a fibrinogen solution containing plasmatic proteins and factor XIII and a thrombin solution with calcium chloride and an antifibrinolytic agent, such as aprotinin. The fibrinogen component, when mixed with thrombin, is converted into polymerizing fibrin monomers. Factor XIII is activated by thrombin in the presence of calcium ions, and the premature lysis of the clot is prevented by aprotinin. Mixing fibrinogen and thrombin simulates the environment of the last stages of the natural coagulation cascade to form a structured fibrin clot similar to a physiological clot, which may be naturally degraded by proteolytic enzymes from the fibrinolytic system, such as plasmin. As a result of its hemostatic and adhesive properties, fibrin glue has been extensively used in Europe in most surgical specialties for over 3 decades to reduce postoperative bleeding, to increase tissue plane adherence, for drug delivery, and in regenerative medicine or tissue engineering [15]. In animal experiments, Pacanowski et al. showed that fibrin glue injection could reduce the strain and pressure transmitted to the aortic aneurysm wall after endovascular exclusion, similar to fresh thrombus [16].

Several studies have reported the safety and effectiveness of fibrin glue for preventing/treating type II endoleaks by the transarterial or direct sac puncture method [11-13,17]. However, the application of fibrin glue to treat type I endoleak has evoked several concerns, namely, the possibility of outflow vessel embolization (such as inferior mesenteric artery and lumbar artery), which may cause ischemia or infarction; the potential for aneurysm thrombosis under systematic pressure; and endoleak recurrence in the long term.

To counter these concerns, we performed angiography to rule out collateral circulation, so all of our patients had pure type I endoleaks. Second, before the injection of fibrin glue, blood flow in the proximal native aorta was blocked by a balloon so that the glue mixture could form a structured fibrin clot and achieve aneurysm sac thrombosis. This procedure prevented embolic clot runoff into a collateral channel. Third, fibrin glue injection in our hands was highly effective in eliminating type I endoleaks, with sustained resolution through a mean 40 months of follow-up. During that time, the mean maximal aneurysm diameter decreased significantly by $9 \mathrm{~mm}$, a strong indicator of durable aneurysm exclusion [18]. Moreover, no fibrin gluerelated complication or mortality was encountered in follow-up.

To monitor the effects of glue injection, intrasac pressure and MPI were measured routinely in our patients. Type I endoleaks were associated with elevated MPI in the majority of cases, which was similar to the data from Dias et al [4]. According to the physical theory Tc $=p R / 2$, tension (Tc) in the aneurysm wall rises with increased intrasac pressure (p) [19]. Comparing pre- and post-embolization intrasac pressures, we found significant pressure reductions after embolization, especially in the pulse pressure, reflecting diminished radial tension in the aneurysm wall.

Intrasac pressure monitoring is still a controversial issue. Some studies have discussed the relationship between intrasac pressure and endoleak changes [14,20], citing these variables as 
markers of successful embolization. Dias et al. found that the pressure varied in different areas of the perigraft space and according to the content within the aneurysm sac, which brought into question the accuracy of the intrasac pressure monitoring [4]. So, for every pressure measurement, we placed the catheter tip at 3 different locations around the endoleak. Moreover, the intrasac pressures, especially the pulse pressure, did indeed decrease in the same measurement location after fibrin glue embolization. Thus, the intrasac pressure measurement, accompanied by final angiography, provided reliable proof of endoleak elimination.

\section{Conclusion of the fibrin glue embolization to treat type I endoleak}

Fibrin glue sac embolization to eliminate type I endoleak after EVAR yielded optimal results in our study, with nearly all the type I endoleaks resolved and no recurrence in follow-up. We believe that fibrin glue embolization may be an ideal option for treating type I endoleak in patients with unfavorable proximal neck anatomy and an alternative to cuff extension with chimney graft rescue of covered renal arteries [21]. Balloon occlusion of the proximal aorta must be done during glue injection to block proximal flow and facilitate formation of a structured fibrin clot. Intrasac pressure monitoring, as well as aortography, appear to be reliable methods of evaluating the effectiveness of fibrin glue sac embolization.

As to prevent the type Ia endoleak on unfavorable aneurysm neck, branched stent-graft, fenestrated stent-graft, or chimney techniques can extend the proximal sealing zone. We believe that this is the trend to decrease the incidence of type Ia endoleak. So in this way, in the last 3 years, the frequency of fibrin glue sac embolization has been significantly reduced.

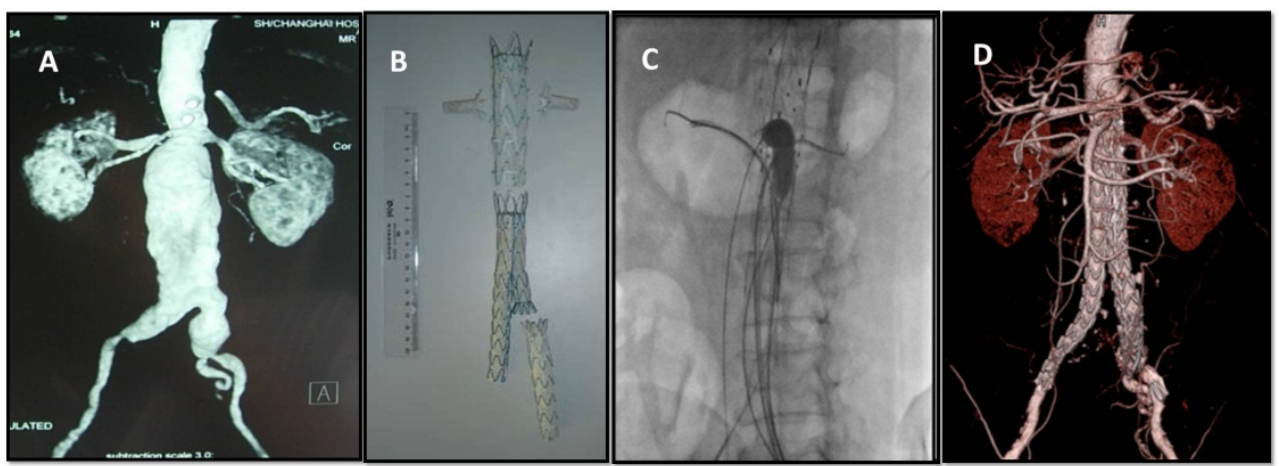

Figure 5. A. Juxta-renal AAA. B. Home-made fenestrated stent-graft of vascular surgery department, Changhai hospital, Shanghai, China. C. Intra-operative alignment. D: 2-year follow-up CTA showed complete exclusion of the AAA, and patent RA and SMA. 

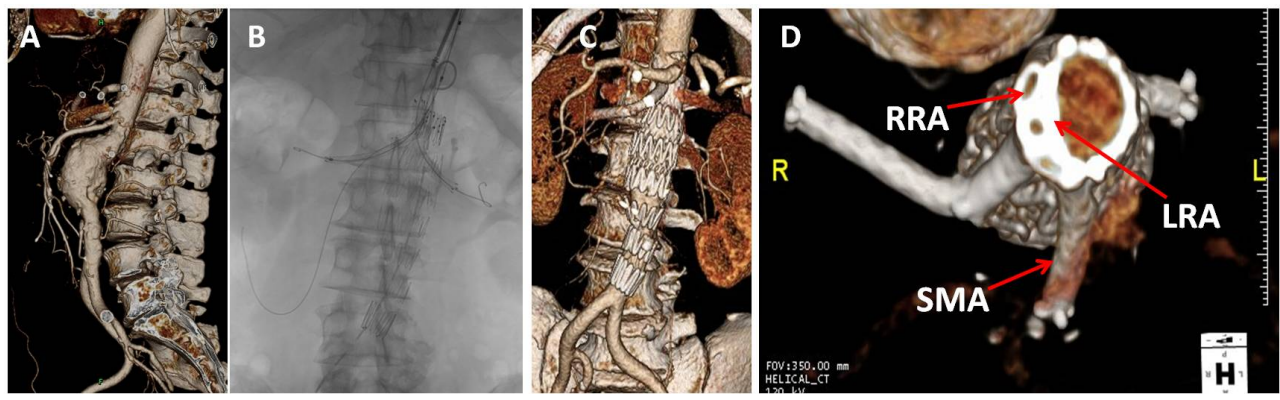

Figure 6. A. Juxta-renal AAA. B. Stenting the RA and SMA before the endografting. C and D: 1-year follow-up demonstrated complete exclusion of the aneurysm and patent branch arteries.

\section{Distal type I endoleak (type Ib endoleak)}

The type Ib endoleak is more common in patients with dilated, calcified, short and tortuous iliac arteries. This can occur when the limb of the graft is too short or migrates upwards due to sac retraction and aortic distortion. Sometimes, when the common iliac artery is short, and the hypogastric artery need to be preserved, distal bare stent can be used to prevent upwards migrates of the iliac extension (Figure 7).
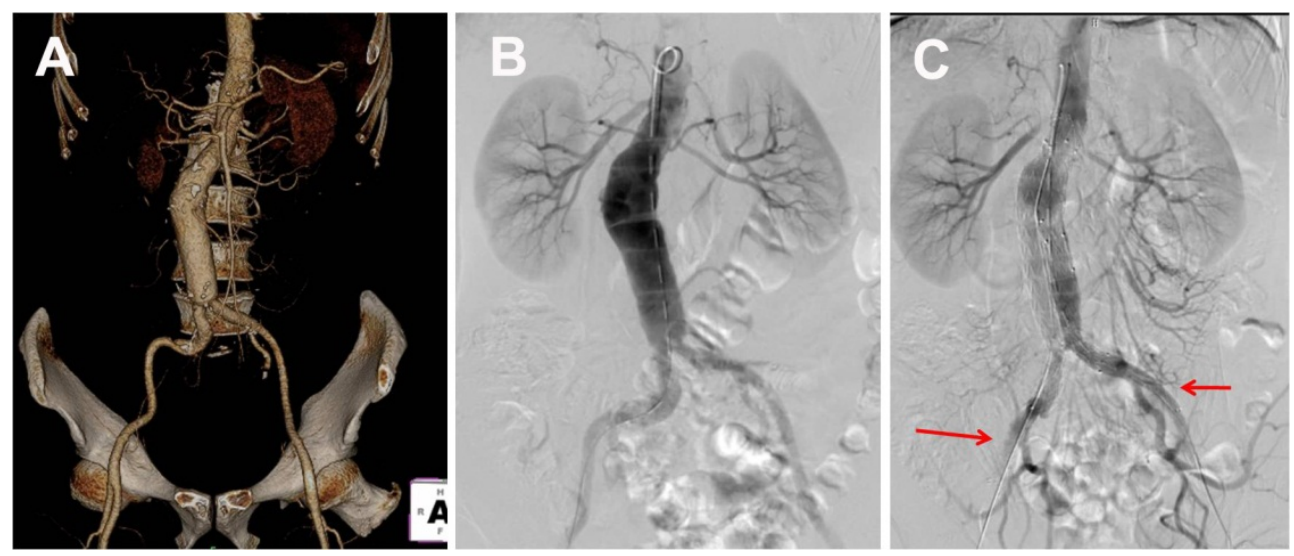

Figure 7. A: $3 D$ reconstruction of pre-operative CTA revealed AAA. The bilateral iliac arteries were in normal diameter. And the common iliac arteries are short. B to C: Distal bare stents were deployed and overlapped with distal extension endograft, to prevent upwards migrates of the iliac extension. 
If both sides of hypogastric arteries are involved by the iliac aneurysm, Iliac Branched Device (IBD) or the Sandwich technique with external iliac artery and internal iliac artery stentgrafting can be used to preserve the one hypogastric artery. And the other one could be occluded. These techniques are proven to be feasible to prevent pelvic ischemic complications and type Ib endoleak (Figure 8).
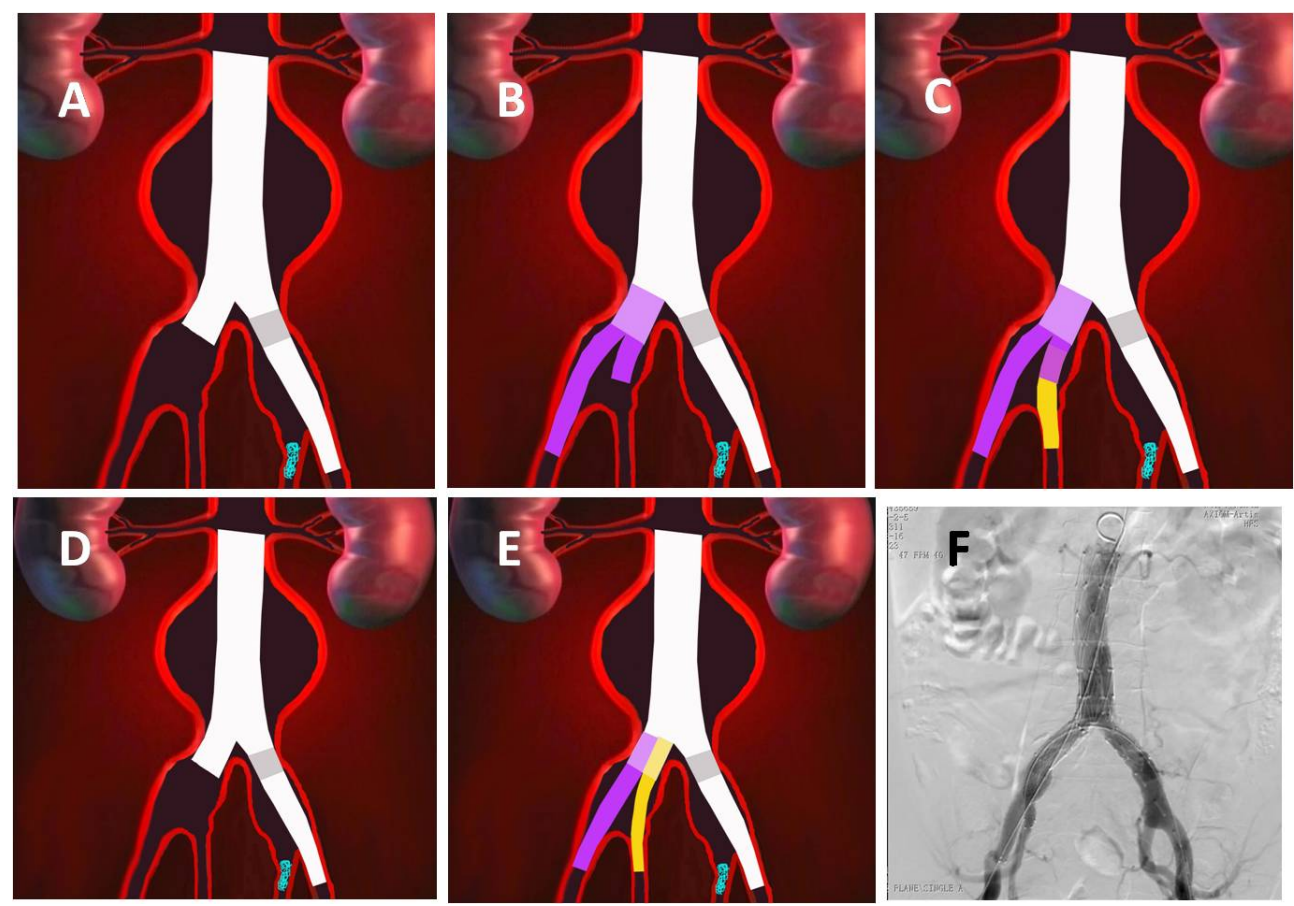

Figure 8. A to C: Iliac Branched Device (IBD) with extension into internal iliac artery. D to F: the Sandwich technique with separated external iliac artery limb and internal iliac artery limb.

Type Ib endoleak is usually treated by distal extension, while distal embolization with microcatheters and glues is less often required as a result of the variety of extender limbs and covered stents available as effective tools. However, sometimes, hypogastric arteries need to be preserved. If required, distal embolization is easier to perform than proximal embolization, as the leak is readily located with a simple curved catheter (Figure 9). 

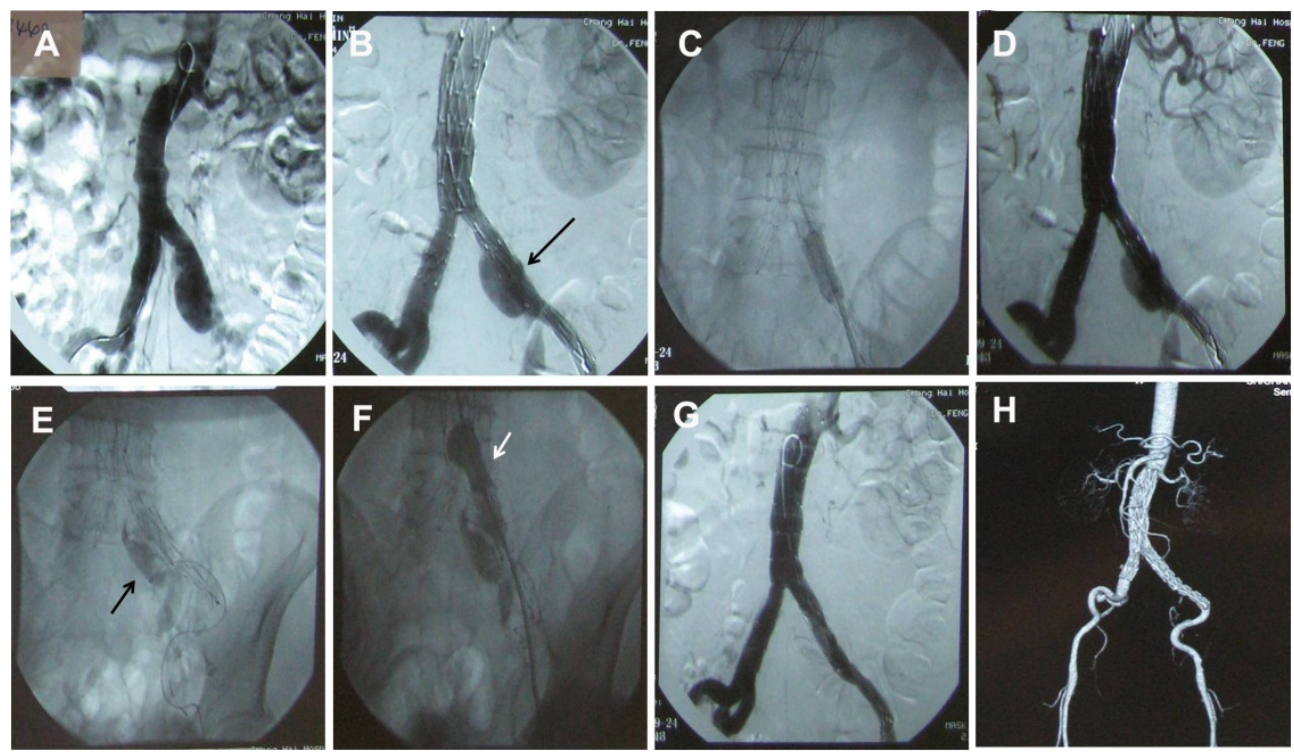

Figure 9. A: Pre-operative angiography revealed AAA (thrombosed) and left common iliac artery aneurysm. B: After bifurcated endografting with left extention were deployed, the type lb endoleak was noted (as black arrow showed). C to D: Ballooning could not diminish the endoleak. E: The distal edge of the extension was probed from the ipsilateral femoral artery. After the tip of the simple curved catheter engaged the channel of leak, a smooth microcatheter could be advanced into this channel. As the black arrow showed, it is essential to pass the microcatheter into the perigraft space and perform angiography to rue out a combined type I and type II endoleak, which is quite common. F: The white arrow showed the proximal ballooning before the embolic agent was injected. G: Final angiography confirmed the satisfying exclusion of the AAA. H: One year follow-up CTA showed no endoleak existed, and the aneurysm was completely excluded.

\section{Type II endoleaks}

Type II endoleak is the most commonly encountered endoleak, and it is much more controversial than type I endoleak. Defined as retrograde flow through collateral vessels into the perigraft space, type II endoleaks prevent thrombosis of the sac and create a potential risk of continued aneurysm expansion and possible rupture. They do not appear to be related to the specific design or material of the endograft used and may appear immediately at the time of graft implantation, at the time of the first follow-up imaging study, or in a delayed fashion months or years after the EVAR procedure [22]. These leaks have also been noted to show resolution and then reappear on subsequent studies. The significance of type II leaks has long been debated, as have appropriate methods of follow-up and determination of the need for intervention. Because at least some of these endoleaks will persist and be associated with aneurysm expansion, there is a need for repeated follow-up imaging studies, which add to the expense of the endovascular approach. There is also the issue of the anxiety engendered in the patient who is uncertain whether his or her aneurysm has been adequately treated. 
The best indicator of hemodynamic significance of a type II endoeak is the associated change in the aneurysm sac: if the sac increases in size, higher pressure and a relatively higher risk of long-term rupture are implied. If the sac is stable or decreasing in size, the risk is likely to be less. An analysis of a large EVAR series found that intervention was necessary only when the sac expanded and that persistent type II endoleaks in the absence of sac expansion could be safely observed [23]. These data are consistent with the earlier recommendations from the EUROSTAR study [5].

After a type II endoleak has been identified on imaging studies, aortography above and within the endograft usually permits exclusion of a type I endoleak and sometimes demonstrates the type II endoleak, particularly on late-phase images. Flush aortography is the starting point but is clearly inadequate to investigate an endoleak, as the majority will not be demonstrated without selective angiography. If the flush study does not show a leak that is present on the imaging study, selective angiography of the superior mesenteric artery and at least one hypogastric artery is performed with prolonged imaging to identify collateral pathways. Specific findings on the imaging study are often suggestive of the source of the leak (eg, left anterolateral blush from the IMA or posterolateral blush for lumbar artery), but may be misleading. The superior mesenteric artery will sometimes fill an IMA endoleak via the middle colic and marginal arteries, but the actual leak may not be demonstrated on main superior mesenteric artery injection. A microcatheter placed superselectively in the marginal artery will be more definitive, showing retrograde filling of the native origin of the IMA with filling of the leak if present. If the marginal artery is of adequate diameter and it is not excessively tortuous, it may be possible to pass the microcatheter back to the origin of the vessel and into the perigraft space. Angiography will then demonstrate the actual size of the leak and demonstrate the direction of flow. Embolization can then be carried out with use of NBCA adhesive, coils, or Onyx co- polymer (Micro Therapeutics, Irvine, California) [24]. Transvascular embolization in the IMA distribution should not be performed if the origin of the vessel cannot be reached, as there is a significant risk of creating colonic ischemia. If a transvascular approach is not possible, the leak can be approached directly by the sac puncture technique described later in the present report. Clipping of the IMA via a laparoscopic approach has also been reported [25].

If the endoleak cannot be reached by transvascular methods, direct puncture of the aneurysm sac with CT, fluoroscopic, or US guidance can be performed [26]. Proper positioning of the catheter in the endoleak cavity is signaled by pulsatile return of blood and opacification of lumbar arteries or IMA on manual injection of contrast. Coils, glue, or thrombin are then deployed until there is no further blood return. There also have been promising results using transcaval embolization for the treatment of type II endoleak. The success rate was reported to be $83 \%$ after one year [27].

\section{Type III endoleaks}

Type III endoleaks arise from inadequate or ineffective seal at the graft junction points between overlapped graft segments or from disconnections and separation of components. Less often 
they are the result of fabric erosion related to material fatigue (Figure 10). Type III endoleak are infrequent, and the incidence was reported to be $4 \%$ after 1 year [28]. Because either of these problems results in arterial flow directly into the perigraft space, they are similar to a type I endoleak and always require intervention. Some failures related to modular dysjunction are preventable by the operator in terms of ensuring adequate overlap of graft components, a parameter that is generally specified by the manufacturer. Extreme angulation of the neck or iliac segments may also be a contributing factor, and may also increase the risk of device migration. Most fabric failures are associated with specific graft materials and designs that have been subsequently modified or withdrawn from the market. Type III endoleaks can generally be treated with deployment of additional stent graft components.
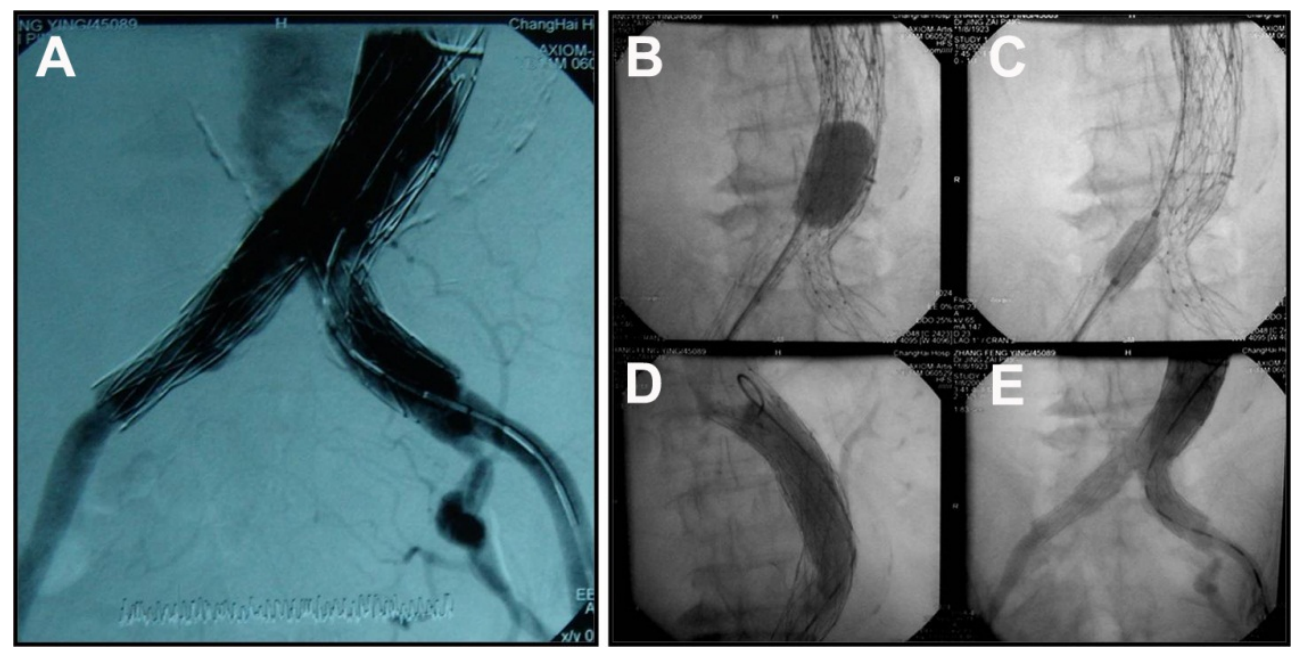

Figure 10. A: Six years after primary EVAR, type III endoleak was identified by routine follow-up CTA. The pre-operative angiography confirmed the graft erosion. B to C: A new one-piece bifurcated endograft was deployed in the previous one, and ballooning was performed. D to E: Aortography above and within the endograft was then performed to confirm the successful exclusion of the endoleak.

\section{Type IV and type V endoleaks}

Type IV endoleaks are not true leaks but represent passage of blood through the graft fabric as a result of porosity. Typically this type of endoleak is transient and only noted at the time of repair appearing as a blush on the post-deployment angiogram, when patients are often fully anticoagulated, and resolve spontaneously after the withdrawal of anticoagulation [29]. This type of endoleak has been eliminated by changes in graft porosity.

Type $\mathrm{V}$ "endoleak" is defined as continued aneurysm sac expansion without a demonstrable leak on any imaging modality. Also referred to as "endotension," this phenomenon remains 
poorly understood, but is likely caused by pulsation of the graft wall, with transmission of the pulse wave through the perigraft space to the native aneurysm wall. It is therefore likely to be related to the graft design, including stent structure and fabric compliance. But we observed persistent pressurization of an aneurysm sac with slow blood flow (slow flow endoleak), which is below the sensitivity limits for detection with current imaging technology. A considerable ultrafiltrate across a microporous fabric can fill the aneurysm and increase the pressure. Endotension seemed more common with expanded ePTFE fabric grafts rather than a woven polyester fabric [30].

Since the source of endotension can be difficult to detect, treatment strategies must be individualized. Relining devices with different low porosity endografts may abolish sac growth or induce sac shrinkage. Occasionally, endograft explantation and conversion to open surgery may be required when no clear cause of endotension can be detected, and endoleak cannot be ruled out (Figure 11).
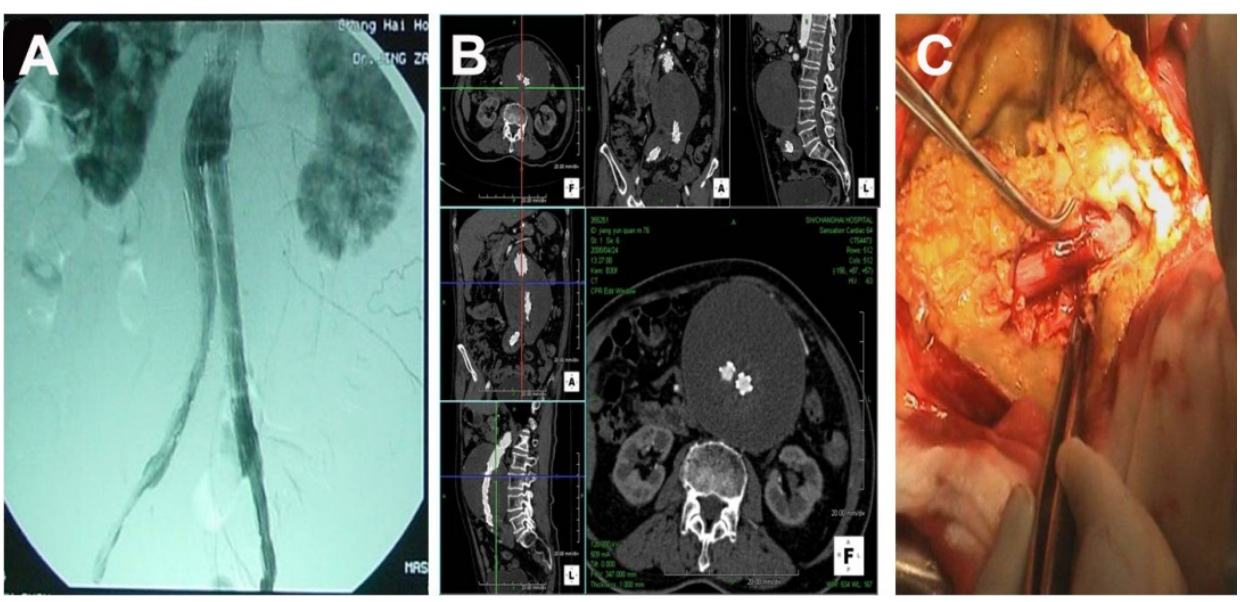

Figure 11. A: Post-operative angiography indicated the successful exclusion of the AAA. B: Four years after the primary EVAR, the aneurysm sac increased in size, without visible endoleak. C: Type $V$ Endoleak was found during the secondary open surgery. The seepage flow and graft erosion was found. When the previous graft was removed and the infra-renal abdominal aorta was reconstructed by a new prosthesis, this patient was successfully treated.

\section{Conclusion words}

Endoleaks continue to be a challenge in the endovascular approach to aneurysm repair. Some of these leaks are device-related and their occurrence has been reduced with continuously improving graft design, whereas type II endoleaks appear to be an intrinsic risk of the endovascular approach. According to our experience, the vast majority of these endoleaks can be treated endoluminally, without resorting to open surgical repair. 
Fibrin glue sac embolization to eliminate type I endoleak after EVAR yielded optimal results in our study. We believe that fibrin glue embolization may be an ideal option for treating type I endoleak in patients with unfavorable proximal neck anatomy and an alternative to cuff extension with chimney graft rescue of covered renal arteries. It is our hope that successful embolization of endoleaks can eliminate or at least reduce the need for lifelong follow-up imaging studies, an important goal in improving patient satisfaction and the economics of the endovascular approach to aneurysm repair. In recent years, the fenestrated stent-graft, branched stent-graft, chimney technique, sandwich technique and Iliac Branched Device, et al, significantly expanded the indication of EVAR, and remarkably reduced the incidence of type I endoleak. We believe that new devices and techniques are the trend to prevent the occurrence of type I endoleak, and finally diminish the usage of glue embolization technique.

In some studies, type II endoleak was aggressively evaluated and treated: if they persist beyond the 6-month study unless there has been shrinkage of the aneurysm sac. But we hold conservative attitude. Persistent type II endoleaks, in the absence of sac expansion, could be safely observed.

\section{Author details}

Zaiping Jing*, Qingsheng Lu, Jiaxuan Feng and Jian Zhou

*Address all correspondence to: xueguanky@163.net

From the Department of Vascular Surgery, Changhai Hospital, Second Military Medical University, Shanghai, China

\section{References}

[1] White, G. H, Yu, W, May, J, et al. Endoleak as a complication of endoluminal grafting of abdominal aortic aneurysms: classification, incidence, diagnosis, and management. J Endovasc Surg. (1997). , 4, 152-168.

[2] Chaikof, E. L, Blankensteijn, J. D, Harris, P. L, et al. Reporting standards for endovascular aortic aneurysm repair. J Vasc Surg. (2002). , 35, 1048-1060.

[3] Veith, F. J, Baum, R. A, Ohki, T, et al. Nature and significance of endoleaks and endotension: summary of opinions expressed at an international conference. J Vasc Surg. (2002). , 35, 1029-1038.

[4] Dias, N. V, Ivancev, K, Resch, T. A, et al. Endoleaks after endovascular aneurysm repair lead to nonuniform intra-aneurysm sac pressure. J Vasc Surg. (2007). , 46, 197-203. 
[5] Van Marrewijk, C, Buth, J, Harris, P. L, et al. Significance of endoleaks after endovascular repair of abdominal aortic aneurysm: the EUROSTAR experience. J Vasc Surg. (2002). , 35, 461-473.

[6] Rosen, R. J, \& Green, R. M. Endoleak management following endovascular aneurysm repair. J Vasc Interv Radiol. (2008). , 19, 37-43.

[7] Faries, P. L, Cadot, H, Agarwal, G, et al. Management of endoleak after endovascular aneurysm repair: cuffs, coils, and conversion. J Vasc Surg. (2003). , 37, 1155-1161.

[8] Kato, N, Semba, C. P, \& Dake, M. D. Embolization of perigraft leaks after endovascular stent-graft treatment of aortic aneurysms. J Vasc Interv Radiol. (1996). , 7, 805-811.

[9] Maldonado, T. S, Rosen, R. J, Rockman, C. B, et al. Initial successful management of type I endoleak after endovascular aortic aneurysm repair with n-butyl cyanoacrylate adhesive. J Vasc Surg. (2003). , 38, 664-670.

[10] Grisafi, J. L, Boiteau, G, Detschelt, E, et al. Endoluminal treatment of type IA endoleak with Onyx. J Vasc Surg. (2010). , 52, 1346-9.

[11] Zanchetta, M, Faresin, F, Pedon, L, et al. Fibrin glue aneurysm sac embolization at the time of endografting. J Endovasc Ther. (2005). , 12, 579-582.

[12] Pilon, F, Tosato, F, Danieli, D, et al. Intrasac fibrin glue injection after platinum coils placement: the efficacy of a simple intraoperative procedure in preventing type II endoleak after endovascular aneurysm repair. Interact Cardiovasc Thorac Surg. (2010). , 11, 78-82.

[13] Meyer, F, Ricke, J, Pech, M, et al. Impressive closure of a sustaining periprosthetic endoleak (type II) using fibrin glue application after former endovascular placement of an infrarenal aortic prosthesis. Int J Surg. (2009). , 7, 84-86.

[14] Baum, R. A, Carpenter, J. P, Cope, C, et al. Aneurysm sac pressure measurements after endovascular repair of abdominal aortic aneurysms. J Vasc Surg. (2001). , 33, 32-41.

[15] Clark, R. A. Fibrin glue for wound repair: facts and fancy. Thromb Haemost. (2003). , 90, 1003-1006.

[16] Pacanowski, J. P, Stevens, S. L, Freeman, M. B, et al. Endotension distribution and the role of thrombus following endovascular AAA exclusion. J Endovasc Ther. (2002). , 9, 639-651.

[17] Ronsivalle, S, Faresin, F, Franz, F, Rettore, C, Zanchetta, M, \& Olivieri, A. Aneurysm sac "thrombization" and stabilization in EVAR: a technique to reduce the risk of type II endoleak. J Endovasc Ther. (2010). , 17, 517-24.

[18] Houbballah, R, Majewski, M, \& Becquemin, J. P. Significant sac retraction after endovascular aneurysm repair is a robust indicator of durable treatment success. J Vasc Surg. (2010). , 52, 878-83. 
[19] Baxendale, B. R, Baker, D. M, Hutchinson, A, et al. Haemodynamic and metabolic response to endovascular repair of infrarenal aortic aneurysms. Br J Anaesth. (1996). , $77,581-585$.

[20] Mehta, M, Veith, F. J, Ohki, T, et al. Significance of endotension, endoleak, and aneurysm pulsatility after endovascular repair. J Vasc Surg. (2003). , 37, 842-846.

[21] Ohrlander, T, Sonesson, B, Ivancev, K, et al. The chimney graft: a technique for preserving or rescuing aortic branch vessels in stent-graft sealing zones. J Endovasc Ther. (2008). , 15, 427-432.

[22] Sheehan, M. K, Ouriel, K, Greenberg, R, et al. Are type II endoleaks after endovascular aneurysm repair endograft dependent? J Vasc Surg. (2006). , 43, 657-61.

[23] Bernhard, V. M, Mitchell, R. S, Matsumura, J. S, et al. Ruptured abdominal aortic aneurysm after endovascular repair. J Vasc Surg. (2002). , 35, 1155-62.

[24] Martin, M. L, Dolmatch, B. L, Fry, P. D, \& Machan, L. S. Treatment of type II endoleaks with Onyx. J Vasc Interv Radiol. (2001). , 12, 629-32.

[25] Zhou, W, Lumsden, A. B, \& Li, J. IMA clipping for a type ii endoleak: combined laparoscopic and endovascular approach. Surg Laparosc Endosc Percutan Tech. (2006). , 16, 272-5.

[26] Baum, R. A, Carpenter, J. P, Golden, M. A, et al. Treatment of type 2 endoleaks after endovascular repair of abdominal aortic aneurysms: comparison of transarterial and translumbar techniques. J Vasc Surg. (2002). , 35, 23-9.

[27] Mansueto, G, Cenzi, D, Scuro, A, et al. Treatment of type II endoleak with a transcatheter transcaval approach: results at 1-year follow-up. J Vasc Surg. (2007). , 45, 1120-7.

[28] Wilt, T. J, Lederle, F. A, Macdonald, R, et al. Comparison of endovascular and open surgical repairs for abdominal aortic aneurysm. Evid Rep Technol Assess. (2006). , 144, $1-113$.

[29] Kanaoka, Y, Ohki, T, Huang, J, et al. A comparison between standard and high density Resilient AneuRx in reducing aneurysm sac pressure in a chronic canine model. J Vasc Surg. (2009). , 49, 1021-8.

[30] Haider, S. E, Najjar, S. F, Cho, J. S, et al. Sac behavior after aneurysm treatment with the Gore Excluder low-permeability aortic endoprosthesis: 12-month comparison to the original Excluder device. J Vasc Surg. (2006). , 44, 694-700. 
